\title{
Overexpression of the UGT73C6 alters brassinosteroid glucoside formation in Arabidopsis thaliana
}

Sigrid Husar ${ }^{1}$, Franz Berthiller², Shozo Fujioka ${ }^{3}$, Wilfried Rozhon ${ }^{1}$, Mamoona Khan ${ }^{1}$, Florian Kalaivanan ${ }^{1}$, Luisa Elias ${ }^{4}$, Gillian S Higgins ${ }^{4}$, Yi Li ${ }^{4}$, Rainer Schuhmacher ${ }^{2}$, Rudolf Krska ${ }^{2}$, Hideharu Seto ${ }^{3}$, Fabian E Vaistij ${ }^{4}$, Dianna Bowles ${ }^{4}$ and Brigitte Poppenberger ${ }^{1, *^{*}}$

\begin{abstract}
Background: Brassinosteroids (BRs) are signaling molecules that play essential roles in the spatial regulation of plant growth and development. In contrast to other plant hormones BRs act locally, close to the sites of their synthesis, and thus homeostatic mechanisms must operate at the cellular level to equilibrate BR concentrations. Whilst it is recognized that levels of bioactive BRs are likely adjusted by controlling the relative rates of biosynthesis and by catabolism, few factors, which participate in these regulatory events, have as yet been identified. Previously we have shown that the UDP-glycosyltransferase UGT73C5 of Arabidopsis thaliana catalyzes 23-O-glucosylation of BRs and that glucosylation renders BRs inactive. This study identifies the closest homologue of UGT73C5, UGT73C6, as an enzyme that is also able to glucosylate BRs in planta.

Results: In a candidate gene approach, in which homologues of UGT73C5 were screened for their potential to induce BR deficiency when over-expressed in plants, UGT73C6 was identified as an enzyme that can glucosylate the BRs CS and BL at their 23-O-positions in planta. GUS reporter analysis indicates that UGT73C6 shows over-lapping, but also distinct expression patterns with UGT73C5 and YFP reporter data suggests that at the cellular level, both UGTs localize to the cytoplasm and to the nucleus. A liquid chromatography high-resolution mass spectrometry method for BR metabolite analysis was developed and applied to determine the kinetics of formation and the catabolic fate of BR-23-O-glucosides in wild type and UGT73C5 and UGT73C6 over-expression lines. This approach identified novel BR catabolites, which are considered to be BR-malonylglucosides, and provided first evidence indicating that glucosylation protects BRs from cellular removal. The physiological significance of BR glucosylation, and the possible role of UGT73C6 as a regulatory factor in this process are discussed in light of the results presented.

Conclusion: The present study generates essential knowledge and molecular and biochemical tools, that will allow for the verification of a potential physiological role of UGT73C6 in BR glucosylation and will facilitate the investigation of the functional significance of BR glucoside formation in plants.
\end{abstract}

Keywords: arabidopsis brassinosteroids, glycosylation, homeostasis, malonylation, steroids

\section{Background}

Brassinosteroids (BRs) are a family of steroid hormones that regulate cell division and cell elongation in plants and participate in the control of growth and development [1]. BRs are synthesized from the sterol campesterol, which is modified by a cascade of hydroxylation and oxidation reactions to yield the biologically active

\footnotetext{
* Correspondence: brigitte.poppenberger@univie.ac.at

'Max F. Perutz Laboratories, University of Vienna, Dr. Bohr-Gasse 9, 1030

Vienna, Austria

Full list of author information is available at the end of the article
}

BRs castasterone (CS) and brassinolide (BL) [2]. CS and BL bioactivity is conferred by their ability to bind to the BR-receptor BRI1 [3], which initiates a phosphorylationdependent signal transduction cascade leading to nuclear acquisition of transcription factors that regulate the expression of BR-responsive genes [4].

Whereas the last decade has seen rapid progress in the identification and characterization of factors, which control BR biosynthesis and participate in BR signal transduction, fewer advances were made in identifying proteins, which directly regulate BR cellular homeostasis.

\section{Biomed Central}


Different homeostatic mechanisms are thought to operate to maintain a BR equilibrium, including the feedback inhibition of BR production [5]. In addition, catabolic inactivation is also considered to play a role in the regulation of bioactive BR levels [2]. CS and BL are catabolically altered or conjugated, with some modifications yielding inactive products. Hydroxylation is one means of catabolic inactivation and is catalyzed by the Arabidopsis thaliana cytochrome P450 monooxygenase BAS1 [6]. Another class of BR conjugates, which are inactive, are glucosides. CS and BL were found to be glucosylated at different positions in feeding studies, with species-specific variations in BR-glucoside profiles [7]. In A. thaliana the hydroxyl groups C-2 and C-23 of CS and BL were identified as target sites for an attachment of glucose $[7,8]$. Whilst enzymes mediating C-2 glucosylation of BRs are still unknown, we could previously show that 23-Oglucosylation of CS and $\mathrm{BL}$ in $A$. thaliana is catalyzed by UGT73C5, a UDP-glycosyltransferase (UGT) [8]. An increase in BR-23-O-glucosylation activity in $U G T 73 C 5$ over-expressing plants correlated with reduced levels of typhasterol (TY), 6-deoxocastasterone (6-deoxoCS) and CS and with BR-deficient phenotypes, showing that 23-O-glucosylation reduces BR bioactivity [8].

UGTs are glycosyltransferases (GTs) of family 1 in the CAZy classification of carbohydrate-active enzymes [9] and catalyze the transfer of glycosyl donor groups to small molecule acceptors, which include secondary metabolites, biotic and abiotic toxins and plant hormones [10,11]. UGTs are regio- and stereo-selective, but are often capable in vitro of recognizing common features on multiple substrates [12]. Moreover, from studies in the multigene family of UGTs in A. thaliana, it has become clear that in vitro a single substrate may be accepted by many individuals of the family $[11,12]$. UGT73C5 has evolved from UGT subfamily 73C [13], which consists of seven UGTs, six of which are clustered in a tandem repeat, are highly similar in their sequences and are promiscuous in their substrate acceptance in vitro. For example, UGT73C6 has been reported as a flavonoid-7-O-glycosyltransferase [14] and is in vitro also capable of conjugating hydroxycoumarins [12], the isoflavone daidzein, the stilbene trans-resveratrol [15], the xenobiotics hydroxylaminodinitrotoluene and aminodinitrotoluene [16], as well as in yeast the fungal toxin zearalenone [17]. Whereas activities of UGT73C subfamily members have been analyzed against various substrates in vitro $[13,15,16,18]$ the in planta substrate specificities and the physiological roles of these UGTs are, with the exception of UGT73C5, as yet little defined.

This study extends and completes the analysis of the UGT73C cluster in regard to the potential of its members to glucosylate BRs and identifies UGT73C6 as a second UGT, which can accept BRs as substrates in planta. It is shown that over-expression of UGT73C6 induces BR-deficient phenotypes, whereas an overexpression of the UGTS 73C1, 73C2, $73 C 3$ and $73 C 4$ does not cause such effects. BR metabolite profiles and BR glucosylation activity analyses provide evidence that UGT73C6 can catalyze CS and BL 23-O-glucosylation in planta. This work also introduces a liquid chromatography high-resolution mass spectrometry (LC-HRMS) method, developed for the detection of BR metabolites, and used as a tool to determine the kinetics of BR-23$O$-glucoside formation in wild type, UGT73C5oe and UGT73C6oe plants. The analysis uncovered the existence of novel BR catabolites, which are considered to be BR-malonylglucosides. LC-HRMS of the kinetics of BL uptake and catabolism in UGT73C5oe and UGT73C6oe lines as compared to wild type provided first indications that glucosylation protects $\mathrm{BL}$ from cellular removal.

\section{Results}

\section{Over-expression of UGT73C6 results in BR deficiency in} A. thaliana

UGT73C5 is a member of UGT subfamily $73 \mathrm{C}$, which is comprised of seven genes, six of which are clustered in a tandem repeat on Chromosome 2 (Figure 1a). The genes of the cluster are highly similar to each other, suggesting that they have evolved from a gene duplication from one ancestral gene and may therefore have related enzymatic properties [19].

Earlier work, which focused on UGT73C5, had demonstrated that constitutive over-expression led to strong BR-deficient phenotypes [8]. Thus, it was of interest to investigate the phenotypic consequences of over-expressing other members of the UGT73C gene cluster. 15 to 25 independent transgenic lines expressing each of the UGTS $73 C 1,73 C 2,73 C 3,73 C 4$ and $73 C 6$ under control of the constitutive Cauliflower Mosaic Virus 35 S (CaMV35S) promoter were generated and analyzed for steady-state levels of transcripts using semi-quantitative RT-PCRs. 3 to 5 lines with high expression levels were then chosen for each UGT to assess effects on plant growth and development. Whereas plants over-expressing the UGT73C1, UGT73C2, UGT73C3 and UGT73C4 did not show any obvious morphological phenotypes (data not shown), an elevated expression of UGT73C6 resulted in drastic growth defects indicative for BR deficiency. As shown in Figure 1bUGT73C6 over-expressing (UGT73C6oe) plants were characterized by dark-green leaves with short petioles and a cabbage-like morphology, delayed flowering and senescence and reduced fertility; these phenotypes correlated in severity with the amounts of UGT73C6 transcript present. 


\section{(a)}

\section{Chromosome II}

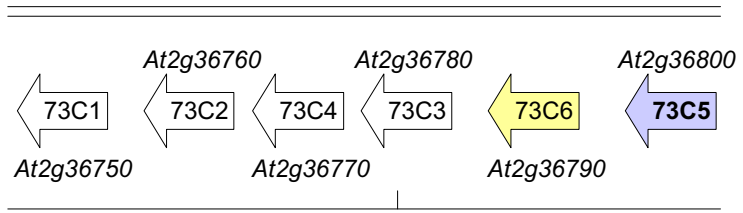

(b)
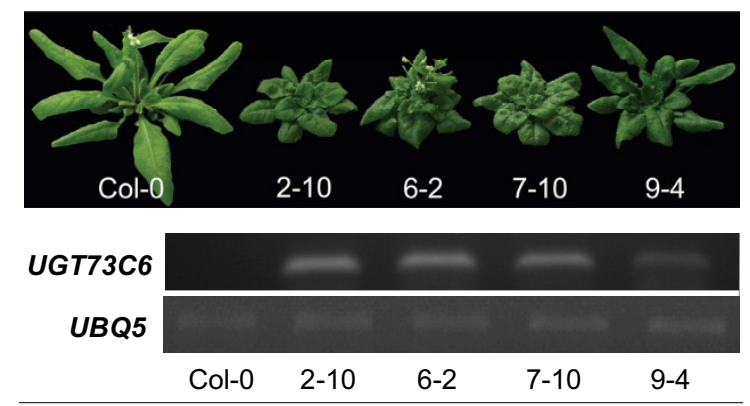

(c)

\begin{tabular}{lcc} 
BRs & $\begin{array}{c}\text { Col-0 } \\
\text { ng/g fw }\end{array}$ & $\begin{array}{c}\text { UGT73C6oe } \\
\text { ng/g fw }\end{array}$ \\
\hline 6-DeoxoCT & $1.29 / 1.08$ & $1.33 / 0.78$ \\
CT & n.d./n.d. & n.d./n.d. \\
6-DeoxoTE & $0.06 / 0.03$ & $0.07 / 0.02$ \\
6-Deoxo3DT & $0.36 / 0.17$ & $0.46 / 0.21$ \\
TE & n.d./n.d. & n.d./n.d. \\
6-DeoxoTY & $0.78 / 0.84$ & $0.62 / 0.82$ \\
TY & $0.24 / 0.23$ & $0.14 / 0.14$ \\
6-DeoxoCS & $1.44 / 1.83$ & $0.94 / 1.28$ \\
CS & $0.60 / 0.50$ & $0.31 / 0.25$ \\
BL & n.d./n.d. & n.d./n.d.
\end{tabular}

Figure 1 Characterization of UGT73C6 over-expressing lines. (a) Illustration of the UGT73C gene cluster. (b) Adult phenotypes of independent transgenic lines expressing a $35 S_{\text {pro: }}$ UGT73C6 construct as compared to wild type Col-0, grown for 4 weeks in long-day conditions (16 hrs $80-100 \mu \mathrm{mol} \cdot \mathrm{m}^{-2} \cdot \mathrm{s}^{-1}$ white light/ $8 \mathrm{hrs} \mathrm{dark)}$ at $21 \pm$ $2^{\circ} \mathrm{C}$. Semi-quantitative RT PCR analysis of UGT73C6 transcript levels in the lines whose phenotype is shown. UBQ5 served as an internal control. (c) BR contents in UGT73C6oe plants as compared to wild type. BR contents were quantified by GC-MS in two independent experiments in which aerial tissues of $A$. thaliana plants, grown in the same conditions as in (a) for $24 \mathrm{~d}$, were compared. BR levels in $\mathrm{ng} / \mathrm{g}$ fw are shown. nd, not detected (below the limit of detection).

To verify if the phenotypic indications for BR deficiency were correlated with changes in endogenous BR levels, BR amounts were analyzed in aerial plant parts of a line with strong UGT73C6 expression (UGT73C6oe) 2-10) and compared to wild type by GC-MS in two independent biological experiments. The results are illustrated in Figure 1c and show that concentrations of TY, 6-deoxoCS and CS were reduced in UGT73C6oe plants. BL was below the limit of detection in both UGT73C6oe and wild type plants.

Taken together these results show that over-expression of UGT73C6 induced phenotypes indicative of impaired BR action in A. thaliana, which correlated with reduced levels of late pathway intermediates of BR biosynthesis.

\section{UGT73C6 catalyzes 23-O-glucosylation of CS and BL in planta}

UGT73C6 has previously been characterized as a UDPglucose:flavonol-3-O-glycoside-7-O-glucosyltransferase, based on a decrease in quercetin-3-O-rhamnoside-7-Oglucoside accumulation in flowers of a UGT73C6 knockout (UGT73C6ko) line and a respective catalytic activity in vitro [14]. To investigate the possibility that UGT73C6, in addition to its role in quercetin-3-O-rhamnoside glucosylation, can also catalyze BR glucosylation in planta, it was anticipated to analyze BR glucoside formation in plants altered in UGT73C6 expression. For this purpose a LC-HRMS method was developed, which is outlined in the experimental procedures section. As reference standards CS-2-O-glucoside (CS-2Glc), CS3-O-glucoside (CS-3Glc), CS-22-O-glucoside (CS-22Glc), $\mathrm{CS}-23-\mathrm{O}$-glucoside (CS-23Glc), BL-2-O-glucoside (BL-2Glc), BL-3-O-glucoside (BL-3Glc), BL-22-O-glucoside (BL-22Glc) and BL-23-O-glucoside (BL-23Glc) were used. The identification was based on retention times and mass spectra, by direct comparison of standards and metabolites. Recovery rates for all measured analytes were between $83 \%$ and $93 \%$ (except for CS with $63 \%$ recovery), with a repeatability ranging from $1.8 \%$ to $3.9 \%$.

Preliminary experiments showed that, in accordance with previous studies $[7,8]$, endogenous BR glucosides were below the limit of detection in untreated plants, also with the newly developed LC-HRMS method. Thus BR glucoside formation was investigated in plants treated with CS or BL. Ten-day-old light-grown seedlings of wild type and UGT73C5oe plants, as well as UGT73C6oe and UGT73C6ko plants, were incubated in media containing either CS or BL for 48 hrs and metabolites formed were measured by LC-HRMS. The results of the feeding studies showed that in plants over-expressing the UGT73C6, in correspondence with plants overexpressing UGT73C5, CS-23Glc and BL-23Glc formation was strongly increased (Table 1) whereas CS-2Glc and BL-2Glc levels appeared unaltered (data not shown). In seedlings of UGT73C6ko plants no statistically significant differences in BR glucosylation activities to wild type were found. Interestingly CS-23Glc and BL-23Glc were not only present in plant extracts, but were also detected in the media, in which the plants had been incubated for the feeding studies (Table 1). 
Table 1 Glucosides of BRs measured in seedlings of UGT73C5oe, UGT73C6oe and wild type used in BR feeding studies

\begin{tabular}{lccc}
\hline & Plant line & $\begin{array}{c}\text { Plant extracts } \\
\mathbf{n g} / \mathbf{g ~ F w}\end{array}$ & $\begin{array}{c}\text { Media } \\
\text { ng }\end{array}$ \\
\hline BL-23Glc & Wild type & $310.8 \pm 77.3$ & $4.4 \pm 2.2$ \\
& UGT73C5oe & $1402.5 \pm 361.7$ & $46.0 \pm 18.4$ \\
& UGT73C6oe & $1489.1 \pm 103.5$ & $23.6 \pm 3.5$ \\
& UGT73C6ko & $428.2 \pm 68.6$ & $1.1 \pm 0.4$ \\
CS-23Glc & Wild type & $34.0 \pm 8.4$ & $5.3 \pm 3.1$ \\
& UGT73C5oe & $153.7 \pm 61.9$ & $37.1 \pm 7.9$ \\
& UGT73C6oe & $154.6 \pm 37.5$ & $43.2 \pm 10.9$ \\
& UGT73C6ko & $47.1 \pm 9.6$ & $0.7 \pm 0.6$ \\
\hline
\end{tabular}

Standard deviation of three independent biological experiments is shown.

Therefore the results are consistent with the hypothesis that UGT73C6 can catalyze 23-O-glucosylation of $\mathrm{CS}$ and BL in planta.

\section{UGT73C6 promoter GUS activity is developmentally regulated}

To analyze the promoter activity of UGT73C6 in different tissues and developmental stages a GUS reporter lines was constructed in which the GUS gene was expressed under control of the UGT73C6 promoter

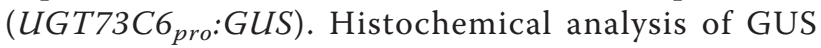
expression in these lines revealed that the UGT73C6 promoter was active in a number of different cell types and was developmentally regulated (Figure 2). Early in development, GUS reporter expression was similar to that previously observed in $U G T 73 C 5_{\text {pro }}$ :GUS plants [20]: a pronounced staining in the vasculature of roots and hypocotyls of young seedlings, both when grown in the light and when incubated in the dark. However, as opposed to UGT73C5 ${ }_{\text {pro }}$ :GUS plants GUS reporter expression in $U G T 73 C 6_{\text {pro }}:$ GUS seedlings was not observed in epidermal cells of the root elongation zone. Later in seedling development, in analogy to UGT73C $5_{\text {pro: }}$ GUS the UGT73C6 pro $_{\text {: }}$ GUS reporter was still active in roots and hypocotyls and moreover was also expressed in cotyledons and true leaves. In contrast to UGT73C5 pro:GUS,

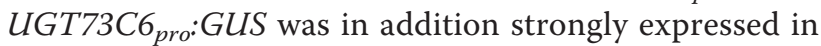
stipules. In flowers $U G T 73 C 6_{p r o}$ :GUS activity was present in sepals and in the stamen filaments. Similar to UGT73C5, UGT73C6 promoter expression was also detected in abscission zones.

In summary there is evidence that the UGT73C6 promoter is subjected to developmental regulation and that it is active in tissues, in which BRs are also known to act. It is worth noting that, when analyzed, the UGT73C6 pro $_{\text {: }}$ GUS reporter was not found to be responsive to externally applied BR (data not shown). To verify this result quantitative real-time PCR (qPCR) analysis of (a)

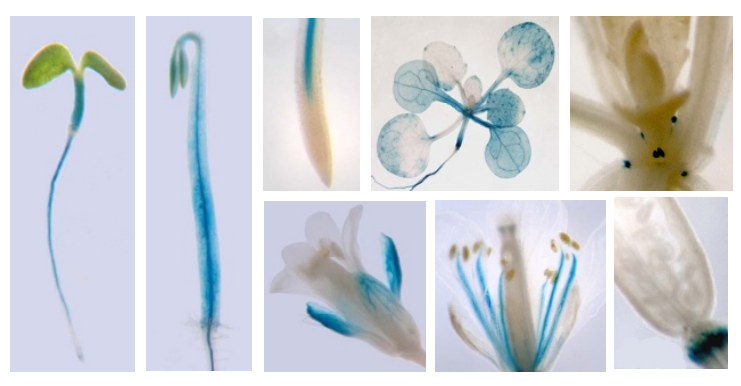

(b)

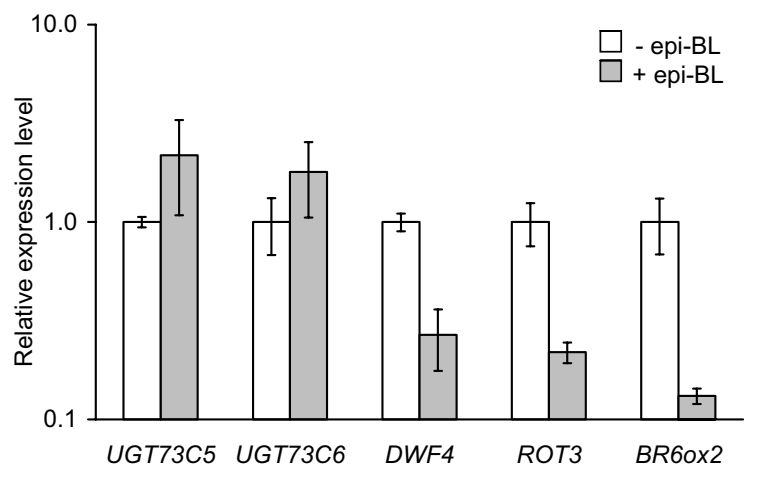

Figure 2 UGT73C6 $6_{\text {pro: }}$ GUS expression is present in all organs and is developmentally regulated. (a) A homozygous line expressing a UGT73C6 promoter GUS fusion, that showed a characteristic staining pattern, was chosen for histochemical analysis of UGT73C6 $6_{\text {pro: }}$ GUS expression in different organs and developmental stages. (b) Response of UGT73C5, UGT73C6, DWF4, ROT3 and BR6ox2 expression in eight-day-old whole seedlings to external application of $1 \mu \mathrm{M}$ 24-epiBL. GAPC2 was used for standardization. The mean and standard deviation of three biological replicates is shown. UGT73C5 and UGT73C6 expression levels are not statistically significantly altered (t-test $p$-value $>0.05$ ) in treated versus untreated samples, while the expression of DWF4, ROT3 and BR60x2 is significantly repressed (t-test p-value $<0.01$ ) by $1 \mu \mathrm{M}$ of 24-epiBL.

8-day old seedlings of wild type, treated with 24-epiBL for $24 \mathrm{hrs}$, was performed. The result showed that in this developmental stage, at 24 hrs post application, 24-epiBL had little effect on UGT73C6 expression on a whole seedling level (Figure 2b). In these conditions also UGT73C5 expression was not significantly altered, whereas DWF4, ROT3 and BR6ox2, genes that are repressed by BR application [5], were significantly decreased in their expression.

\section{Subcellular localization of UGT73C6 expression}

To investigate the cellular sites of UGT73C6 protein localization plants expressing UGT73C6-YFP reporter constructs were generated. For this purpose two vectors were cloned: one in which the YFP-tagged coding sequence of UGT73C6 was placed down-stream of its 
own promoter (UGT73C6 $\left.6_{\text {pro }}: U G T 73 C 6-Y F P\right)$, and another one in which the UGT73C6-YFP fusion was driven by the CaMV35 $S$ promoter. A. thaliana plants stably expressing the two constructs were generated and YFP expression levels were assessed in seedlings of homozygous lines using Western blot analysis. The results are illustrated in Figure $3 \mathrm{a}$ and show that lines 3, 4, 5 and 6 expressed UGT73C6-YFP to high levels; these lines also showed BR-deficient phenotypes, indicating that the UGT73C6-YFP fusion was active.

Imaging of $Y F P$ expression in seedlings of $35 S_{\text {pro }}$ : UGT73C6-YFP and UGT73C6 pro $_{\text {:UGT73C6-YFP lines }}$ using confocal microscopy revealed that UGT73C6-YFP was localized in the cytoplasm, as well as in the nucleus (Figure 3b). As expected fluorescence in $35 S_{\text {pro }}$ : UGT73C6-YFP was stronger than in UGT73C6 $6_{\text {pro: }}$ : UGT73C6-YFP lines, but showed an identical localization pattern. $35 S_{\text {pro }}: U G T 73 C 5-Y F P$ showed the same subcellular localization pattern as $35 S_{\text {pro }}:$ UGT73C6-YFP (data not shown).

To verify the nuclear localization of UGT73C6-YFP the reporter was transiently co-expressed with a CFP fusion of $\mathrm{BES1}$, a protein that is known to localize predominantly to the nucleus [4], in tobacco. The result showed that UGT73C6-YFP co-localized with BES1-CFP (Figure 4) providing evidence that the UGT73C6-YFP reporter, in addition to the cytoplasm also localizes to the nucleus.

\section{Kinetics of BL-23-O-glucoside formation}

To investigate the conversion of $\mathrm{BL}$ into BL-23Glc in UGT73C5oe and UGT73C6oe plants over time, a timecourse feeding study was initiated. Eleven-day-old seedlings of wild type Col-0, UGT73C5oe and UGT73C6oe were incubated with $1 \mu \mathrm{g} / \mathrm{ml}(2.1 \mu \mathrm{M})$ of BL. Samples were harvested in a time-course manner and BL-23Glc formation was determined in tissue extracts by

\section{(a)}

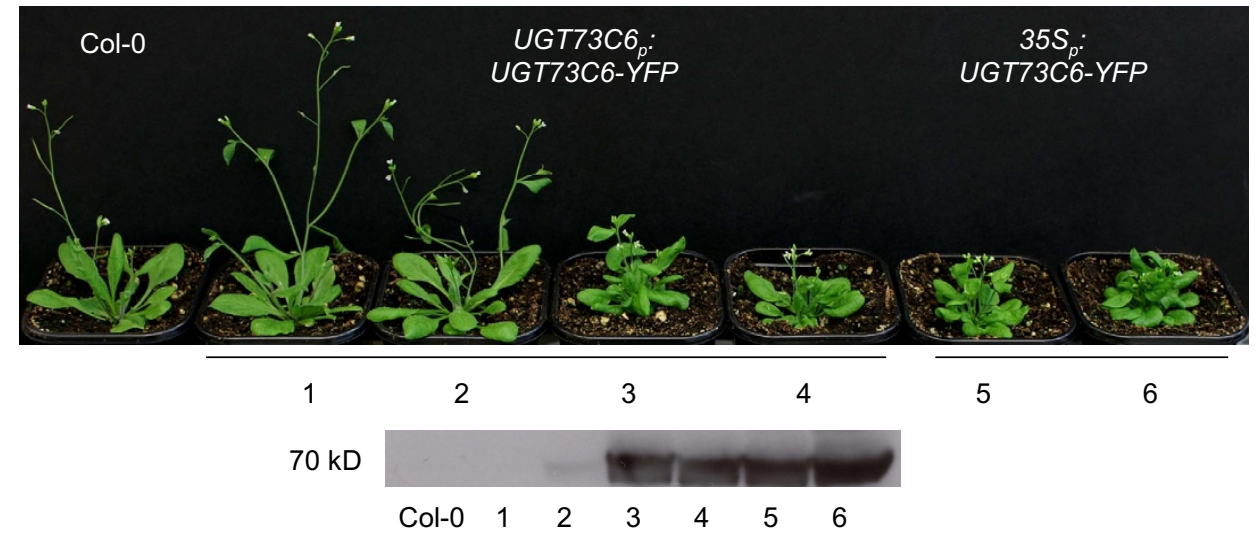

(b)

$35 S_{p}:$ UGT73C6-YFP
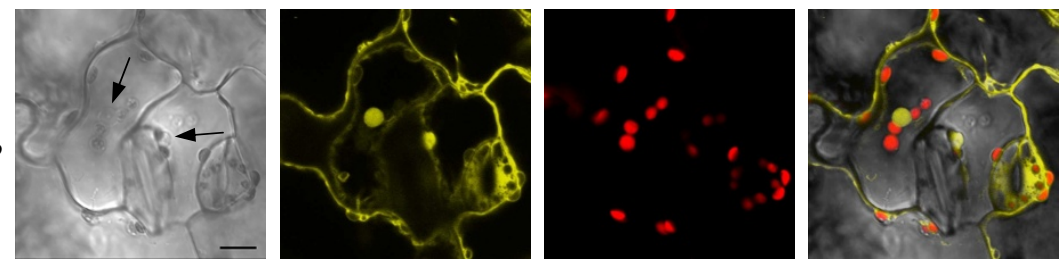

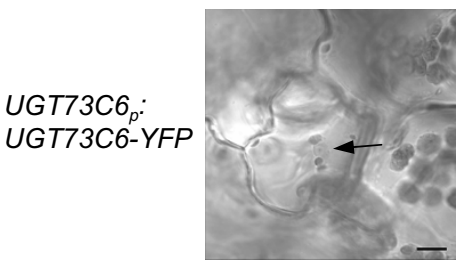

bright field

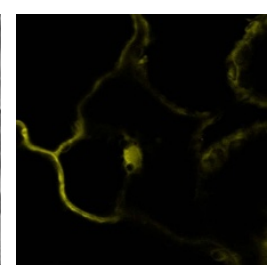

YFP filter

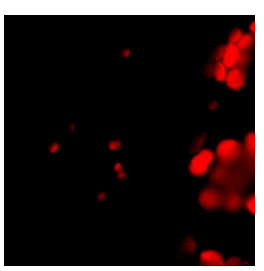

filter

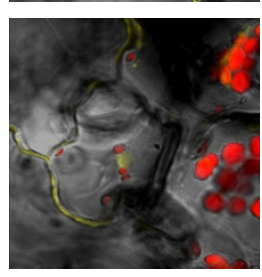

overlay

Figure 3 UGT73C6-YFP reporter generation and analysis. (a) Top: Adult phenotypes of independent transgenic lines expressing either a UGT73C6 $6_{\text {pro }}$ UGT73C6-YFP construct (center) or a 35S pro:UGT73C6-YFP construct (right) as compared to wild type (wt), grown for 4 weeks in longday conditions (16 hrs 80-100 $\mathrm{\mu mol} \cdot \mathrm{m}^{-2} \cdot \mathrm{s}^{-1}$ white light/8 hrs dark) at $21 \pm 2^{\circ} \mathrm{C}$. Bottom: Western blot analysis of UGT73C6-YFP protein levels in 2-week-old seedlings of the lines whose phenotype is shown above, using an anti-GFP antibody. (b) Representative YFP expression pattern of UGT73C6-YFP analyzed in leaves of eleven-day-old seedlings of line 35S pro:UGT73C6-YFP/4 and line UGT73C6 pro:UGT73C6-YFP/5 by fluorescence microscopy. The scale bars represent $10 \mu \mathrm{m}$. 


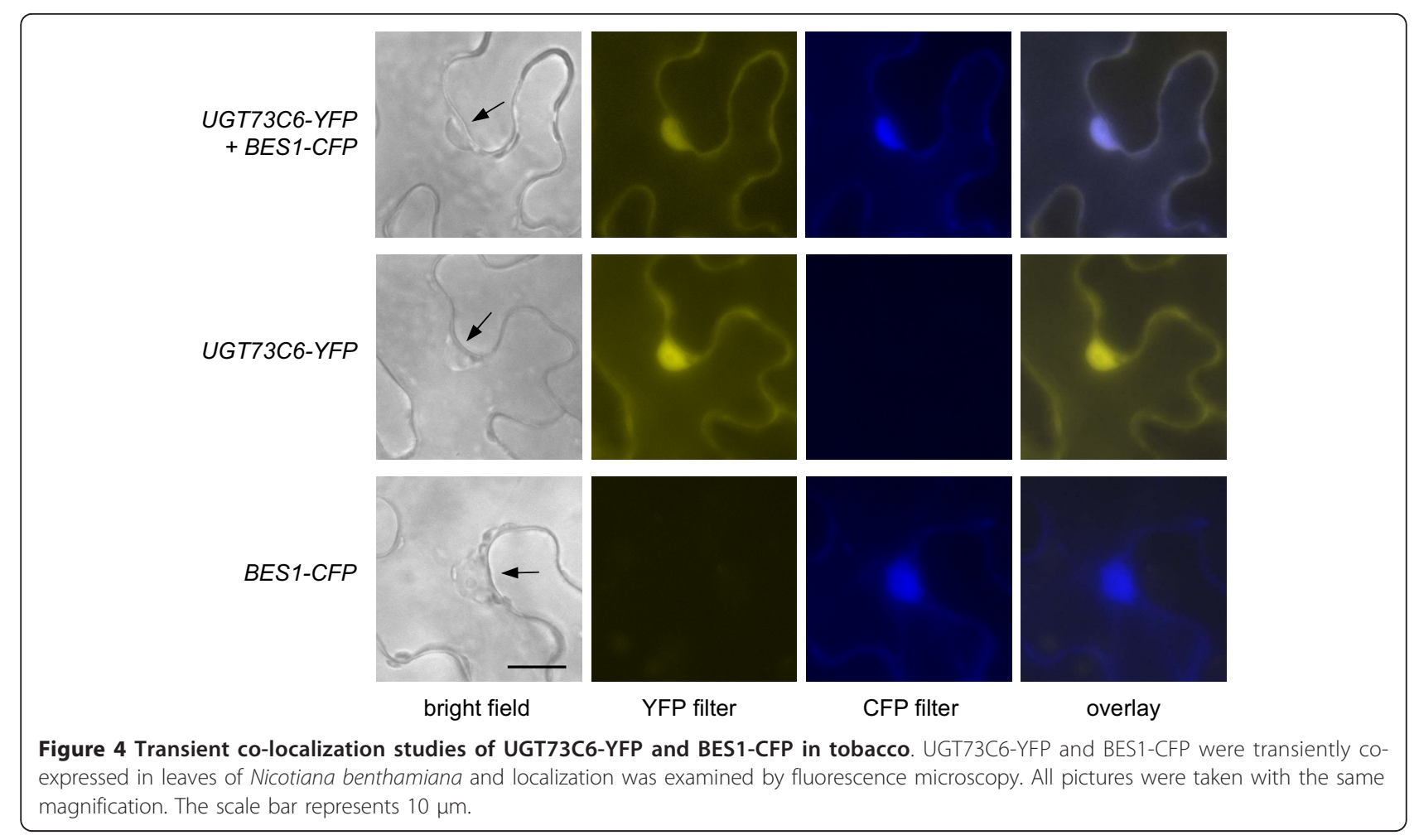

LC-HRMS. As shown in Figure 5a BL was rapidly incorporated, as evidenced by a strong increase in endogenous $\mathrm{BL}$ amounts following $\mathrm{BL}$ application. In wild type seedlings BL levels increased rapidly for $12 \mathrm{hrs}$ following $\mathrm{BL}$ application, before they started to decline. BL levels in UGT73C5oe and UGT73C6oe also increased for approximately $12 \mathrm{hrs}$ post application of BL, however BL amounts only reached about $50 \%$ of the levels, which were accumulated in wild type (Figure 5a). 96 hrs post application, BL levels in both wild type and UGT73C5oe and UGT73C6oe lines had dropped to levels below the limit of detection.

BL-23Glc formation in wild type seedlings slowly increased for $24 \mathrm{hrs}$, before BL-23Glc amounts started to decrease. In UGT73C5oe and UGT73C6oe plants the concentration of BL-23Glc strongly increased for approximately $12 \mathrm{hrs}$, reaching amounts which were approximately 10 -fold higher, than those measured in wild type (Figure $5 \mathrm{~b}$ ).

In summary exogenously applied BL was rapidly incorporate by both wild type and UGT73C5oe and UGT73C6oe plants and was thereafter efficiently removed. In contrast, following its formation, BL-23Glc was maintained at elevated levels in plant tissues.

\section{Catabolic fate of BR-23-O-glucosides}

The decrease of BL-23Glc levels in plant tissues, starting at $12 \mathrm{hrs}$ post application of BL in UGT73C5oe and
UGT73C6oe seedlings and at $24 \mathrm{hrs}$ in wild type, indicated that the BL-23Glc formed was either immobilized, degraded or was further modified to yield products, which escaped detection. Also, previously it had been shown that in BL-feeding studies of wild type $A$. thali$a n a$, an initial increase in BL-23Glc formation was followed by a decrease, indicating a further metabolization [7]. Thus it was of interest to investigate the catabolic fate of externally applied CS and BL. LC-HRMS was used to analyze BR conjugates in seedlings of wild type, UGT73C5oe and UGT73C6oe plants, following $48 \mathrm{hrs}$ of incubation with either CS or BL. In addition to significant amounts of BR-23Glc, minor peaks corresponding to BR-2Glc, BR-sulfate and BR-hydroxide were found. Moreover, very interestingly, a previously unknown substance with a mass of $\mathrm{m} / z 751.3877$ eluted at $9.61 \mathrm{~min}$ (compared to $9.54 \mathrm{~min}$ of BL-23Glc), in seemingly high abundance, from the column (Figure 6). According to accurate mass measurements the compound was tentatively identified as BL-malonylglucoside (BL-MalGlc). The theoretical mass of the sodium adduct of this substance is 751.3875 (0.2 ppm deviation), the only possible sum formula is $\mathrm{C}_{37} \mathrm{H}_{60} \mathrm{O}_{14}$ (subtracting the sodium adduct; nitrogen rule applied; max. 1 ppm mass deviation; max. 10 nitrogen, 30 oxygen, 100 carbon and 200 hydrogen atoms). As malonylglucosides are formed from glucosides it is highly likely that the compound is BL-23-O-malonylglucoside (BL-23MalGlc). 


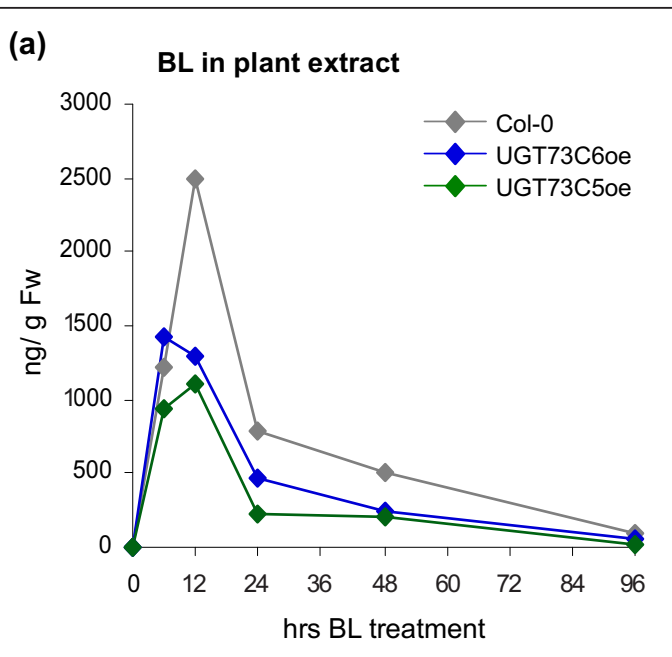

(b) BL-23-O-glucoside in plant extract

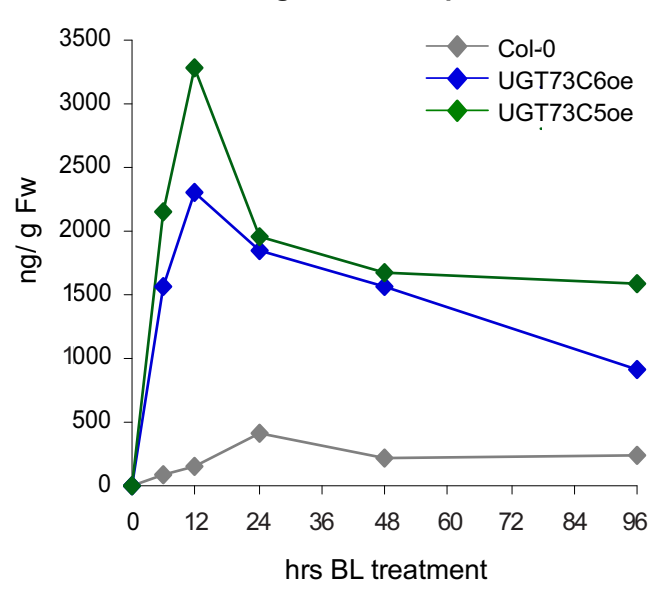

Figure $5 \mathrm{BL}$ and BL-23Glc levels formed in seedlings of A. thaliana used in BL feeding studies over time, analyzed by LC-HRMS. eleven-day-old seedlings were incubated for the indicated periods of time in ATS media supplemented with $30 \mu \mathrm{g}$ $B L$, and $B L$ contents were quantified from plant extracts by LCHRMS analysis. (a) BL and (b) BL-23Glc levels are shown in ng/g Fw.

Similarly, as shown in Figure 7, when plants fed with CS were analyzed for CS-catabolites a peak at $10.29 \mathrm{~min}$ (compared to $10.25 \mathrm{~min}$ of CS-23G), showing a $\mathrm{m} / \mathrm{z}$ of 735.3929, appeared and was tentatively identified as CS-malonylglucoside (CS-MalGlc). Only one sum formula is conceivable when applying the criteria outlined above, namely $\mathrm{C}_{37} \mathrm{H}_{60} \mathrm{O}_{13}$ (mass deviation $0.4 \mathrm{ppm}$ ). Again, it seems highly likely that the substance is CS-23-O-malonylglucoside (CS-23MalGlc). In addition to the putative BR-MalGlcs BR-diglucosides (BR-diGlc) were also identified. Interestingly, both the formation of the putative BR-MalGlcs and the BR-diglucoside was increased in UGT73C6oe and UGT73C5oe seedlings as compared to those of wild type indicating that they were formed from BR-23Glcs (Figure 8). In analogy to

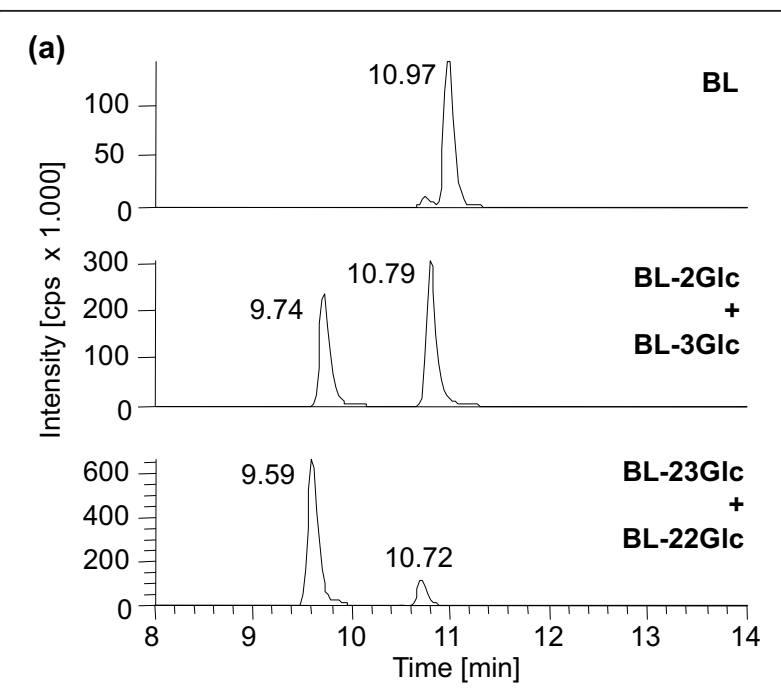

(b)

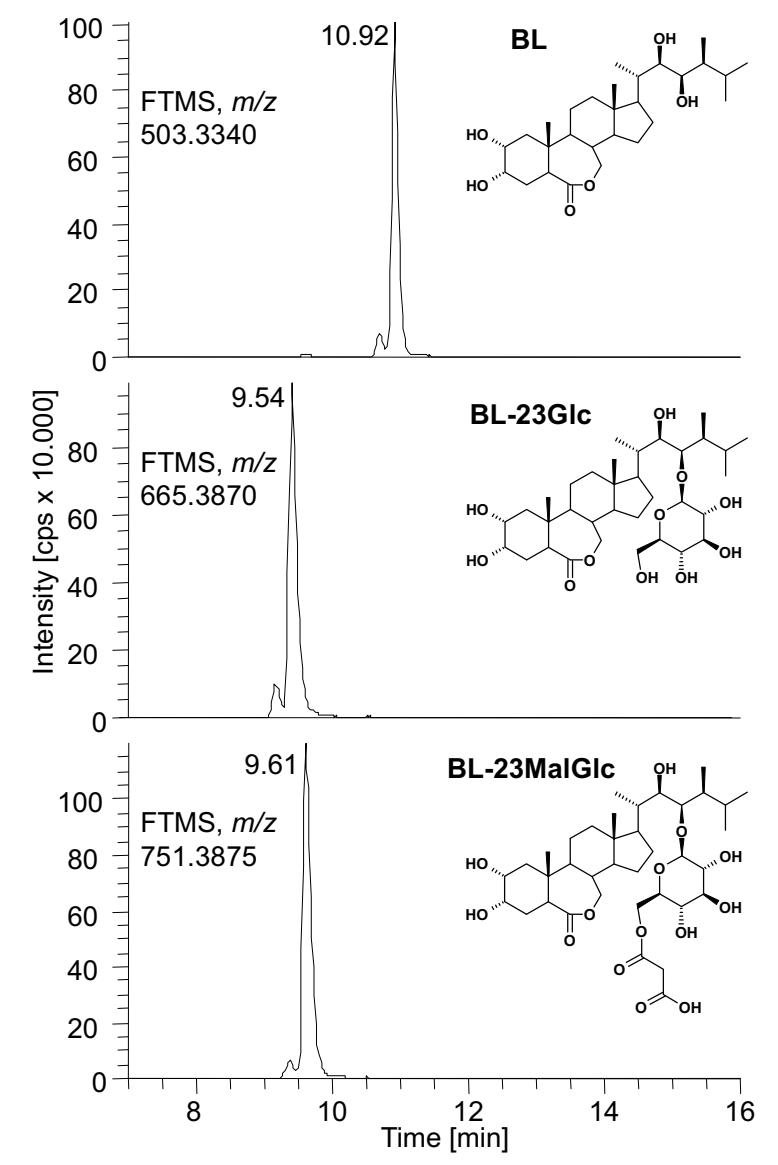

Figure 6 Identification of a novel BL-Glc catabolite. HR-LCMS analysis was employed to identify glucosides formed in BL feeding experiments of seedlings over-expressing UGT73C5. (a) HR-LCMS mass chromatograms of authentic BL-O-glucoside standards. (b) HR-LCMS mass chromatograms of metabolites formed in UGT73C5oe seedlings. Theoretical masses of sodium adducts and predicted structures are shown. The position of the malonylgroup in the putative BL-23MalGlc is not certain. 


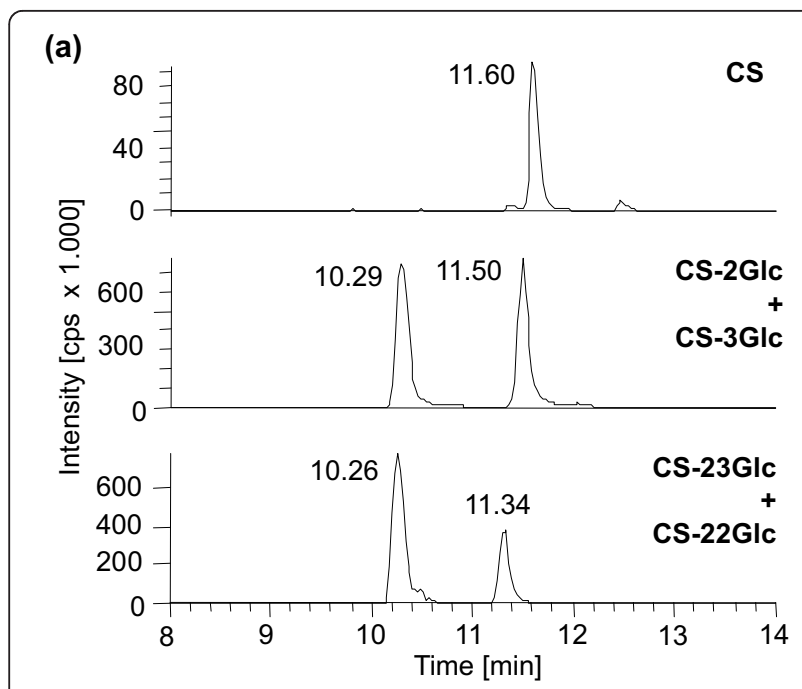

(b)

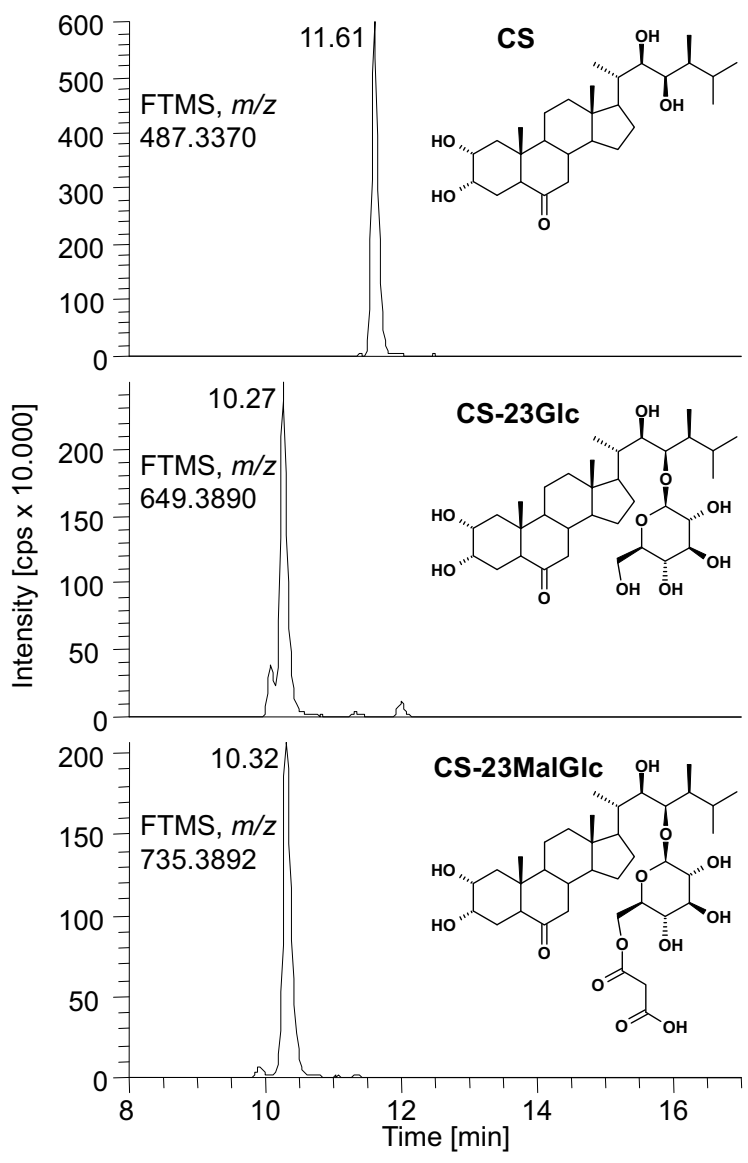

Figure 7 Identification of a novel CS-Glc catabolite. HR-LCMS analysis was employed to identify glucosides formed in BL feeding experiments of seedlings over-expressing UGT73C5. (a) HR-LCMS mass chromatograms of authentic CS-O-glucoside standards. (b) HR-LCMS mass chromatograms of metabolites formed in UGT73C5oe seedlings. Theoretical masses of sodium adducts and predicted structures are shown. Please note that the position of the malonyl group in the putative CS-23MalGlc is not certain.

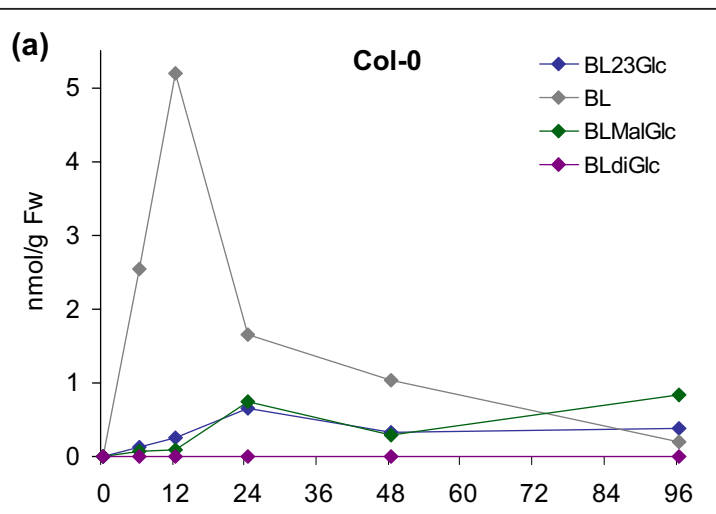

(b)

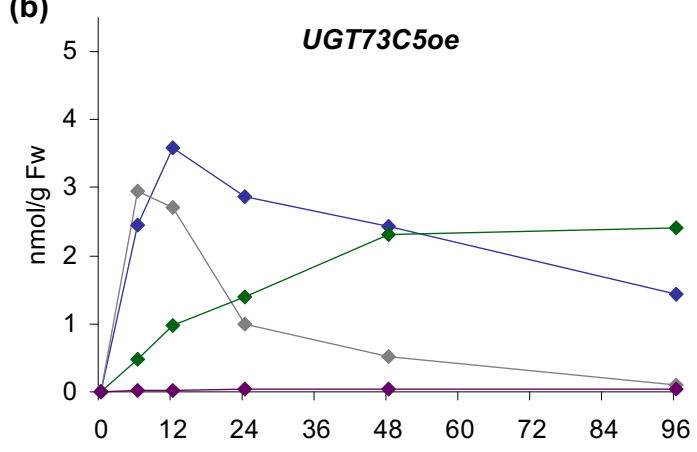

(c)

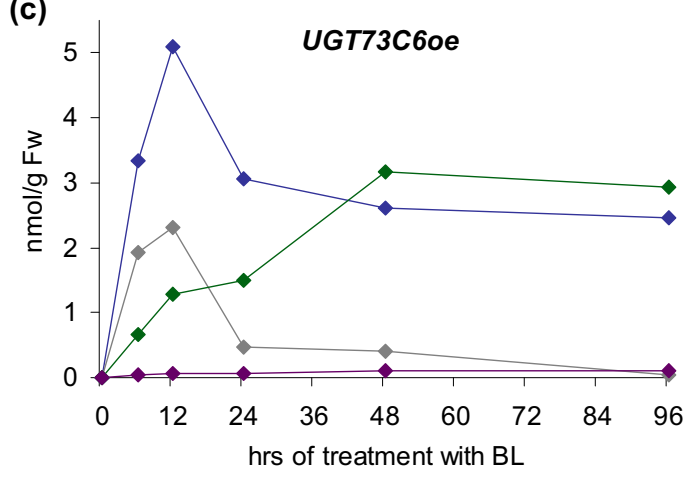

Figure 8 Analysis of wild type and UGT73C5oe and UGT73C6oe seedlings, used in BL feeding studies, for the occurrence of BL-MalGlc over time. The values shown are nmol/g Fw.

BR-23Glc both BR-diGlc and the putative BR-MalGlc were not only detected in plant extracts, but were also present in the media in which plants had been incubated for the feeding studies (data not shown); thus BR-Glcs formed in planta were released to the media.

In summary these results suggest that 23-O-glucosides of $\mathrm{BL}$ and CS are further modified by malonylation in planta.

\section{Kinetics of BL-glucoside catabolism in UGT73C5oe and UGT73C6oe plants}

To determine the kinetics of formation of the putative BR-MalGlc and BR-diGlc, the samples of the time- 
course BL feeding studies were analyzed for an occurrence of BL-23Glc catabolites. At present no analytical standard is available for BL-MalGlc to accurately quantify its amounts. However, as a rough estimate the same response factor as for BL-23Glc was assumed, allowing for a semi-quantitative estimation of BL-MalGlc concentrations. Similarly the concentration of BL-diGlc was estimated by assuming the same response factor as for BR-23Glc. The results are illustrated in Figure 8 and show levels of BL-MalGlc and BL-diGlc in nmol/g Fw, as compared to $\mathrm{BL}$ and $\mathrm{BL}-23 \mathrm{Glc}$ amounts in seedlings of Col-0, UGT73C5oe and UGT73C6oe. All BL-Glcs detected were present in all analyzed lines, however in wild type BL-diGlc was close to the limit of detection with the applied LC-HRMS method. Amounts of the putative BL-MalGlc increased in wild type for $12 \mathrm{hrs}$ and were then sustained (Figure 8a). Similarly the kinetics of putative BL-MalGlc formation in UGT73C5oe and UGT73C6oe lines were characterized by an increase to a plateau concentration within 48 hrs of feeding, which was then sustained for the rest of the experiment (Figure 8b, c). This is in contrast to BL and BL-23Glc levels, which decreased in both wild type and UGT73C5oe and UGT73C6oe after having reached a peak. Interestingly, a drop in BL-23Glc amounts correlated with a corresponding increase in putative BL-MalGlc in UGT73C5oe and UGT73C6oe plants, supporting the notion that BL-23Glc was converted to BL-23MalGlc.

In summary the results show that in BL feeding studies of wild type, and UGT73C5oe and UGT73C6oe plants a decrease in BL-23Glc levels correlated with an increase in putative BL-MalGlc, showing that BL-23Glc was further conjugated. As opposed to BL and BL-23Glc the putative BL-MalGlc did not decrease after an initial increase, suggesting that malonylation may protect 23O-glucosylated BL from removal, in the soluble fractions analyzed.

\section{Discussion}

Glycosylation is considered an important regulatory mechanism that contributes to the control of hormone homeostasis and almost all major classes of hormones occur as glycoside-conjugates in planta [10,11,21]. BRs are one class of plant hormones, which are glycosylated $[2,7]$ and previously we have shown that conjugation to glucose reduces BR activity. Over-expression of UGT73C5 led to a massive increase in BR-23-O-glucosylation activity and to decreased levels of bioactive BRs, evidenced both at the chemotypic and at the phenotypic level [8]. UGT73C5 belongs to a cluster of six closely related genes in the A. thaliana genome, UGT73C1-C6 [13]. The in vitro catalytic activities of the six gene products have been characterized to some extend, and it appears that members of the UGT73C subfamily can recognize a number of aglycons including secondary metabolites, plant hormones, fungal mycotoxins and xenobiotics as substrates in vitro $[12,15,16,18,20,22]$. However, nothing was known of the consequences of over-expressing the five remaining members of the $73 \mathrm{C}$ gene cluster UGT73C1-C4 and UGT73C6 on plant growth and development and in particular also on those growth processes regulated by BRs. This study was designed to investigate those consequences, aiming at identifying possible functional homologues of UGT73C5, and revealed that UGT73C6, the closest homologue of UGT73C5, can also accept BRs as substrates in planta.

Overexpression of UGT73C6 led to the same phenotypic effects as observed in UGT73C5oe plants: growth defects indicative of BR deficiency. Moreover at the chemotypic level UGT73C6oe plants were characterized by significantly reduced amounts of TY, 6-deoxoCS and $\mathrm{CS}$, which correlated with a strongly increased 23-O-glucosylation activity in CS and BL feeding studies. These data show that in planta UGT73C6 can catalyze 23-Oglucosylation of CS and BL and is likely also able to glucosylate TY and 6-deoxoCS. Interestingly, UGT73C6 was previously identified as a flavonol-3-O-glycoside-7$O$-glucosyltransferase [14] and was in vitro capable of recognizing an array of structurally highly diverse aglycons [12,14-16]. Thus the question arose if, in addition to its role in quercetin-3-O-rhamnoside conjugation, UGT73C6 may also catalyze BR glucosylation in planta. To try to answer this question seedlings of a UGT73C6ko line were analyzed for alterations in BR-23$O$-glucosylation activities, but no significant change in the formation of CS- and BL-23Glcs were found. This result can be interpreted in several ways. First, it is possible that the endogenous gene UGT73C6 does not function in BR-23-O-glucosylation in planta. Second, the expression and function of UGT73C6 may be highly specific to particular cells or developmental events, and the impact of losing its activity was not observed under the conditions assayed in this study. Third, UGT73C5 or other GTs that glucosylate BRs in planta, and are coordinately regulated, may complement for a loss of UGT73C6 function and thus, knocking out UGT73C6 only will not produce a phenotype. Indeed functional redundancy is characteristic of regulatory events governing BR action $[4,23]$ and has also been shown to play a role in BR catabolism: the cytochrome P450 monooxygenase SOB7 acts redundantly with BAS1 in the inactivation of BRs [24]. To refine UGT73C6 function in the context of functional redundancy it was therefore aimed to generate plants deficient in the expression of both UGT73C6 and UGT73C5. However, several approaches including the use of RNAi [25] and artificial microRNAs [26], failed in generating double knock-down plants. 
Thus, in summary we provide evidence that UGT73C6 is capable of glucosylating BRs in planta, however at present we cannot answer the question if BR glucosylation is also a physiological function of UGT73C6. Further work will be needed to address this issue.

The expression of UGT73C6 was analyzed at the subcellular and cellular level and it was found that UGT73C6 shows over-lapping, but also distinct expression/localization patterns with UGT73C5. GUS reporter data suggests that transcript abundance of both genes is developmentally regulated and is enriched in vascular tissues, which are also tissues in which genes involved in BR biosynthesis are preferentially expressed $[27,28]$. At the transcriptional level, in seedlings, UGT73C6 expression was not found to be responsive to externally applied BR. However, interestingly UGT73C6 mRNA levels are increased in response to a large variety of stimuli including (a) toxins of exogenous and endogenous origin such as the mycotoxin deoxynivalenol [20], the explosive TNT [16], the herbicide imidazolinone [29], as well as the allelochemical benzoxazolin-2(3H)-one [30] and oligogalacturonides released from plant cell walls by pathogen polygalacturonases [31], and (b) abiotic and biotic stress factors such as salt stress [32,33] and Botrytis cinerea infections [34]. Therefore UGT73C6 has been proposed to comprise a component of a co-ordinately regulated, broad specificity, xenobiotic defense response machinery [30]. UGT73C5 shows a similar responsiveness to toxins in its transcriptional regulation [20] and it will thus be interesting to determine how responsiveness to abiotic and biotic stimuli is coordinated with the putative functions of UGT73C5 and UGT73C6 in glucosylating BRs and/or flavonols.

On a cellular level YFP reporter data indicate that UGT73C5 and UGT73C6 localize to the cytoplasm and intriguingly also to the nucleus. In mammals, where there is considerable interest in UDP-glucuronyltransferases as regulators of metabolic homeostasis, it is thought that, in addition to cytoplasmatic functions, UGTs may also act in the nucleus to control the steady state of ligands for nuclear receptors and protect nuclear components from toxins $[35,36]$. In this context the UGT2B7, which glycosylates steroid hormones, retinoids, fatty acids as well as xenobiotics, has been shown to be present and active both in the ER and in the nucleus [37]. Also plant UGTs have previously been found to exhibit dual subcellular localizations [38], however the functional significance is as yet unknown.

Another so far unresolved question is the function of BR-Glc formation. Whereas it is well documented that glycosylation can alter the bioactivity of plant hormones including auxins, cytokinins, abscisic acid and gibberellins the reason why glycoside conjugates are inactive is unclear $[39,40]$. In principle glycosylation could inhibit hormone activity directly by interfering with receptor recognition or indirectly by inducing events, which are enabled by the glycosylation status [40]. In this context, glycosylation is known to facilitate transport and results of this study indicate that also BR glycosides are transported, either actively or passively. Glycosylation is also considered to alter the stability of aglycons [41] and here first evidence is presented which indicates that 23-O-glucosylation protects BRs from degradation and/ or catabolism. Moreover it is shown for the first time that CS- and BL-23Glcs are further conjugated, likely by malonylation.

Malonylation is an aliphatic acylation, which involves a regiospecific malonyl group transfer from malonylCoA to the glycosyl moiety of a glycoside, and is catalyzed by acyltransferases of the BAHD family $[42,43]$. Malonylation modifies secondary metabolites such as flavonoids, isoflavonoids, anthocyanins and terpenoids and is considered to enhance solubility, protect glycosides from enzymatic degradation by glycosidases and facilitate their intracellular transport [42,43]. Malonylation has also been implicated in the regulation of hormone homeostasis. The ethylene precursor ACC can be irreversibly conjugated to form $N$-malonyl-ACC [44] and thus malonylation of ACC decreases the levels of ethylene in producing tissues. In regard to BR catabolism the results of this study show that the putative BL-MalGlc formed in UGT73C5oe and UGT73C6oe lines is less readily removed from soluble cellular fractions than BL-23Glc, indicating that malonylation may serve to protect BL-23Glc from catabolism or degradation by enzymes such as glucosidases. In this context it will be interesting to determine if de-glucosylation is a means of reactivating BRs from BR-Glcs and thus, if BR-Glcs may serve as readily available BR storage forms.

\section{Conclusions}

In summary this study provides evidence that in addition to UGT73C5, also its closest homologue UGT73C6, is able to catalyze 23-O-glucosylation of the bioactive BRs CS and BL in planta. Future studies will address the question, if BR glucosylation is a physiological role of both UGTs, and if this potential multiplicity may provide a highly flexible system for homeostatic adaptation at a cellular level.

\section{Methods}

\section{Plant material and growth conditions}

A. thaliana ecotype Columbia- 0 (Col-0) was used as the wild type for all experiments carried out in this study. For phenotypic analysis, if not indicated differently, plants were cultivated in a growth room with long-day growth conditions (16 hrs white light, $80-100 \mu \mathrm{mol} \cdot \mathrm{m}^{-}$ ${ }^{2} \cdot \mathrm{s}^{-1} / 8 \mathrm{hrs}$ dark) at $21 \pm 2^{\circ} \mathrm{C}$. Plant transformation and 
seed sterilization was performed as described previously [45]. ATS media [46] was used for plant growth under sterile conditions.

\section{Chemicals}

BL and CS were purchased from Synthchem Inc. (Waterloo, Ontario, Canada). 24-epiBL was obtained from Sigma-Aldrich (St. Louis, USA). Stock solutions of $100 \mu \mathrm{g} / \mathrm{mL}$ in ethanol were made and stored in amber screw vials at $-20^{\circ} \mathrm{C}$. Synthesis of 2-O-, 3-O-, 22-O- and 23-O-Glcs of BL and CS will be described elsewhere (Seto, unpublished). The BR-Glcs were stored in amber screw vials at $-20^{\circ} \mathrm{C}$ as $50 \mu \mathrm{g} / \mathrm{mL}$ stocks in ethanol. Water for LC was purified using a MilliQ system (Millipore, Molsheim, France). LC gradient grade methanol, acetonitrile and sodium chloride (p.a.) were purchased from Merck (Merck KGaA, Darmstadt, Germany). LCMS grade formic acid was obtained from Sigma-Aldrich (St. Louis, USA). Ethyl acetate was supplied by Carl Roth (Karlsruhe, Germany). Strata Si-1 silica gel SPE cartridges $(500 \mathrm{mg}, 6 \mathrm{~mL}$ ) and security guard C18 precolumns were acquired from Phenomenex (Aschaffenburg, Germany).

\section{Generation of transgenic lines}

For the generation of plants over-expressing individual members of the UGT73C subfamily, the open reading frames of UGT73C1, UGT73C3, UGT73C4 and UGT73C6 were PCR amplified from plasmids containing the corresponding genes [13] and were cloned into a modified version of the binary vector pBIN19 called $\mathrm{pJR} 1 \mathrm{Ri}$, in which expression of the transgenes is driven by the CaMV35 S promoter [47]. UGT73C2 was amplified from genomic DNA with the primer pairs UGT73C2-fw/UGT73C2-rv (for sequences of all primers used see Additional File 1) and was cloned EcoRV and NotI into the binary plant expression vector, pGWR8, which also expresses transgenes under control of the CaMV35 S promoter [48].

Plants of $A$. thaliana were transformed with the representative constructs using the floral dip method [45] and 15-25 independent transgenic lines were selected for each construct. Plants homozygous for the transgenes were then analyzed by semi-quantitative RT-PCR analysis for transcript abundance using gene specific primers and 2-5 lines with high expression levels were chosen for phenotypic analysis.

For the generation of UGT73C6 promoter GUS lines the promoter and the 5' UTR of UGT73C6 (-1687 to +4 relative to the translational start) was PCR amplified from genomic A. thaliana DNA using Taq polymerase (Fermentas, St. Leon-Rot, Germany) and the primer pair 73C6p-GUS-fw/73C6p-GUS-rv, and was cloned into pPZP-GUS.1 using PstI+BamHI [20]. Plants were transformed with the constructs, 20 independent lines were selected and a line with a representative GUS expression pattern was chosen for subsequent analysis.

For the generation of YFP reporter lines the ORFs of UGT73C5 and UGT73C6 were PCR amplified from genomic A. thaliana DNA using Taq polymerase and the primers $73 C 5 \mathrm{cds}-\mathrm{YFP}-\mathrm{fw} / 73 \mathrm{C} 5 \mathrm{cds}-\mathrm{YFP}-\mathrm{rv}$ and 73C6cds-YFP-fw/73C6cds-YFP-rv, and the PCR products obtained were cloned NcoI+NotI into pGWR8 [48] down-stream of the CaMV35 S promoter. YFP was then added in frame to the $C$-terminal parts of the genes to create YFP-fusion constructs. A YFP reporter construct driven by the endogenous UGT73C6 promoter was cloned by PCR, amplifying the 5'UTRs of UGT73C6 from genomic DNA using primers 73C6pYFP-fw/73C6-YFP-rv and replacing the $35 \mathrm{~S}$ promoters with the obtained PCR product. Following plant transformation 10-20 independent transgenic lines were selected for each construct and plants homozygous for the transgenes were analyzed by Western blotting for YFP fusion protein abundance.

\section{Analysis of BR levels using gas chromatography mass spectrometry (GC-MS)}

For BR measurements plants were grown in long-day conditions (16 hrs cool white light, $80-100 \mu \mathrm{mol} \cdot \mathrm{m}^{-2} \cdot \mathrm{s}^{-1}$ / 8 hrs dark) at $21 \pm 2^{\circ} \mathrm{C}$ for 24 days before tissue of aerial plant parts was harvested. Fifty grams (fresh weight) of plant material was lyophilized and extracted twice with $500 \mathrm{ml}$ of $\mathrm{MeOH}: \mathrm{CHCl}_{3}$ (4:1) and deuteriumlabeled internal standards $1 \mathrm{ng} / \mathrm{g}$ fresh weight were added. Purification and quantification of BRs was performed as described previously [49].

\section{Sample preparation for the analysis of metabolism of BL and CS in plants}

Fifty eleven-day-old seedlings of Col-0, UGT73C6oe, UGT73C6ko and UGT73C5oe, grown on ATS plates, were transferred to sterile Erlenmeyer flasks containing $30 \mathrm{ml}$ liquid ATS media and incubated on a shaker $(60$ $\mathrm{rpm})$ in continuous light $\left(80 \mu \mathrm{mol} \cdot \mathrm{m}^{-2} \cdot \mathrm{s}^{-1}\right)$ conditions at $21^{\circ} \mathrm{C} \pm 2^{\circ} .24 \mathrm{hrs}$ after transfer of the plants, BL or CS were added to an end concentration of $1 \mu \mathrm{g} / \mathrm{ml}(2.1$ and $2.2 \mu \mathrm{M}$, respectively) and the seedlings were incubated for the indicated periods of time. The plant material (on average 0.8 to $1.0 \mathrm{~g}$ ) was then harvested, ground in liquid nitrogen and extracted twice with $5 \mathrm{ml}$ aqueous methanol $(50+50, v+v)$. The methanolic plant extracts $(10 \mathrm{ml})$ were dried down under a gentle stream of nitrogen at $40^{\circ} \mathrm{C}$ and re-dissolved in $2.5 \mathrm{ml}$ saturated $\mathrm{NaCl}$ solution and $2.5 \mathrm{ml}$ water. Liquid/liquid extraction was performed 3 times with $5 \mathrm{ml}$ ethyl acetate each. The ethyl acetate phases were combined, dried down under nitrogen and re-dissolved in $1 \mathrm{ml}$ ethyl acetate. Strata 
Si-1 silica gel SPE cartridges were conditioned with $5 \mathrm{ml}$ acetonitrile and equilibrated with $10 \mathrm{ml}$ ethyl acetate before the sample was applied. The cartridges were washed with $5 \mathrm{~mL}$ ethyl acetate, removing most of the chlorophyll. The BRs and their glucosides were eluted with $5 \mathrm{ml}$ of ethyl acetate/methanol 20/80 (v/v), dried under nitrogen and reconstituted in $1 \mathrm{ml} 70 \%$ methanol for analysis by LC-HRMS. For the time course experiment samples were taken before and 6, 12, 24, 48 and $96 \mathrm{hrs}$ after addition of BL $(1 \mu \mathrm{g} / \mathrm{ml})$.

\section{Liquid chromatography high-resolution mass spectrometry (LC-HRMS) for the analysis of BRs and their glucosides}

A LC-HRMS method was developed for the quantification of BRs and BR-Glcs as well as their metabolites produced in plant tissues. An Accela HPLC pump (Thermo Fisher Scientific, Waltham, MA, USA) together with a Mistral column thermostat (Maylab, Thermo Fisher Scientific) and a PAL HTC autosampler (CTC Analytics, Zwingen, Switzerland) were coupled to a LTQ Orbitrap XL high-resolution mass spectrometer (Thermo Fisher Scientific). Separation was performed on a Hypersil Gold column $(150 \times 2.1 \mathrm{~mm}, 3 \mu \mathrm{m}$ particle size; Thermo Fisher Scientific) at $25^{\circ} \mathrm{C}$. Gradient separation used water with $0.1 \%$ formic acid as solvent A and methanol with $0.1 \%$ formic acid as solvent B. $50 \%$ $B$ were kept for $1 \mathrm{~min}$, then a linear gradient reached $100 \%$ B at $12 \mathrm{~min}$. After a 5 min washing step with $100 \% \mathrm{~B}$, the solvent composition was changed back to $50 \% \mathrm{~B}$ within one min and the column was re-equilibrated till the end of the run at $32 \mathrm{~min}$. A divert valve redirected the eluent into the ion source between 8 and $13 \mathrm{~min}$ to minimize unnecessary contamination of the MS. A flow rate of $250 \mu \mathrm{l} / \mathrm{min}$ was chosen, the injection volume was $5 \mu \mathrm{l}$. Ionization was performed in the electrospray positive mode at $300^{\circ} \mathrm{C}$ with the following settings: sheath gas flow 45 , aux. gas flow 5 , source voltage $4 \mathrm{kV}$, capillary voltage $5 \mathrm{~V}$, tube lens $200 \mathrm{~V}$. Centroid FTMS data were acquired from $\mathrm{m} / z$ 200-1000 with a resolution of 60.000 . The sodium adduct of n-butylbenzenesulfonamide (nBBS; $m / z 226.071570$ ) was found to be ubiquitous in our system and was used as lock mass. Instrument control and data evaluation was performed with Xcalibur 2.0.7. For the latter, a mass tolerance of 5 ppm was allowed for the following masses: $[\mathrm{BL}+\mathrm{Na}]^{+} \mathrm{m} /$ $z$ 503.3343; $[\mathrm{CS}+\mathrm{Na}]^{+} \mathrm{m} / z$ 487.3394; [BL-glucosides $\left.+\mathrm{Na}\right]^{+}$ $m / z$ 665.3871; [CS-glucosides+Na] ${ }^{+} m / z$ 649.3922. Retention times were: BL23G: $9.54 \mathrm{~min}$; BL2G: $9.74 \mathrm{~min}$; CS23G: $10.25 \mathrm{~min}$; CS2G: $10.32 \mathrm{~min}$; BL22G: $10.72 \mathrm{~min}$; BL3G: 10.79 min; BL: $10.97 \mathrm{~min}$; CS22G: $11.34 \mathrm{~min}$; CS3G $11.50 \mathrm{~min}$; CS $11.60 \mathrm{~min}$.

External standard calibration was performed with $1 / x$ weighted models. While the parent substances BL and
CS showed highly linear correlations from 1 - $1000 \mathrm{ng} /$ $\mathrm{ml}$, quadratic models were used for all eight glucosides over the same concentration range. Recovery and repeatability was tested by spiking $100 \mathrm{ng} / \mathrm{ml}$ of all analytes in liquid media or methanolic extracts of untreated plants in quadruplicates before clean-up and measurement.

\section{Semi-quantitative and quantitative real-time PCRs}

For semi-quantitative RT-PCRs RNA was isolated from plant material using the RNeasy Plant Mini Kit from Qiagen (Qiagen GmbH, Hilden, Germany) and cDNAs were synthesized with the RevertAid H Minus First Strand cDNA Synthesis Kit (Fermentas) from DNaseItreated RNA. PCRs were performed with specific primers for the UGTs 73C1, 73C2, $73 C 3$ and $73 C 4$ (sequences see Additional File 1) and UGT73C5 [20] and UGT73C6 [8].

For analysis of transcript levels by qPCR five-day-old seedlings, grown on ATS plates, were transferred to sterile Erlenmeyer flasks containing $30 \mathrm{ml}$ of liquid ATS media and incubated on a shaker $(60 \mathrm{rpm})$ in continuous light $\left(80 \mu \mathrm{mol} \cdot \mathrm{m}^{-2} \cdot \mathrm{s}^{-1}\right)$ at $21^{\circ} \mathrm{C} \pm 2^{\circ}$ for $3 \mathrm{~d}$. Subsequently, 24-epiBL (dissolved in DMSO) was added to an end concentration of $1 \mu \mathrm{M}$ and the plants were incubated for another $24 \mathrm{hrs}$ under the same conditions. As a control, seedlings were treated with an equal amount of DMSO. RNA isolation, cDNA sythesis and qPCRs were performed as described previously [50] using the primers listed in Additional File 1. The relative expression levels were calculated from three biological replicates, each measured in technical quadruplicate, after normalization to GAPC2 [51]. The expression levels of treated and untreated samples were considered as statistically significantly different, if the p-value of a two-tailed t-test using $\log _{2}$-transformed results was below 0.05 .

\section{Western blot analysis}

Leaf tissues (100 mg) were ground in liquid nitrogen using a Qiagen TissueLyser II and extracted with $300 \mu \mathrm{l}$ extraction buffer (66 mM TRIS/HCl pH 6.8, $133 \mathrm{mM}$ DTT, $2.7 \%$ SDS, $13 \%$ glycerol, $0.01 \%$ bromophenol blue). $20 \mu \mathrm{l}$ of these extracts were separated by SDSPAGE (10\% gel) and blotted onto Immobilon P (Millipore Cooperation, Bedford, MA, USA). The membranes were first incubated with mouse anti-GFP antibody and second with alkaline phosphatase-conjugated goat antimouse IgG (Santa Cruz Biotechnology, CA, USA). Detection was performed by enhanced chemiluminescence using the CDP-Star detection reagent (Amersham Bioscience, NJ, USA).

\section{Reporter localization analysis}

GUS activity of UGT73C6 pro: GUS lines was analyzed histochemically as described previously [20]. For YFP 
localization studies seedlings were grown on ATS plates [46] for 11 days and were subsequently analyzed with a Zeiss LSM Meta confocal microscope (Zeiss, Oberkochen, Germany). YFP-tagged fusion proteins and chlorplast autofluorescence were both excited using the Ar laser line at $488 \mathrm{~nm}$ and were detected at 530-565 nm (yellow channel) and 625-700 nm (red channel), respectively. The images were assembled using the Zeiss LSM image browser software version 4.2.0.121. For colocalization studies YFP and CFP-tagged versions of UGT73C6 and BES1 [48] were transiently expressed in leaves of Nicotiana bentamiana by infiltration with agrobacteria as described previously [52]. Infiltrated leaves were examined by fluorescence microscopy using a Zeiss Axioplan 2 microscope equipped with a Zeiss AxioCam MRc5 camera.

\section{Additional material}

Additional file 1: Primers used in this study. A table providing the sequences of primers used in this study.

\section{Acknowledgments and Funding}

We would like to thank the horticultural staff of the University of York and the Max F. Perutz Laboratories for excellent plant care and Prof. Kazuki Saito for kindly providing seeds of the UGT73C6ko line. We also thank Dr. Suguru Takatsuto (Joetsu University of Education) for supplying deuterium-labeled internal standards. This work was supported by funds from the Austrian Science Fund FWF, the United Kingdom Biotechnology and Biological Sciences Research Council, the Garfield Western Foundation and by a Grantin-Aid for Scientific Research (B) from the Ministry of Education, Culture, Sports, Science and Technology of Japan to SF (Grant No. 19380069). The LC-MS system was funded by the Federal Country Lower Austria and cofinanced by the European regional development fund of the European Union. MK received a fellowship from the Higher Education Commission of Pakistan; BP received an Erwin-Schrödinger and a Hertha-Firnberg fellowship from the FWF.

\section{Author details}

${ }^{1}$ Max F. Perutz Laboratories, University of Vienna, Dr. Bohr-Gasse 9, 1030 Vienna, Austria. ${ }^{2}$ Center for Analytical Chemistry, Department of Agrobiotechnology, University of Natural Resources and Life Sciences, Konrad Lorenz Straße 20, 3430 Tulln, Austria. ${ }^{3}$ RIKEN Advanced Science Institute, Wako-shi, Saitama 351-0198, Japan. ${ }^{4}$ Center for Novel Agricultural Products, Department of Biology, University of York, York YO10 5DD, UK.

\section{Authors' contributions}

SH carried out the GUS reporter analysis, generated and analyzed YFP reporter lines, generated and analyzed over-expression lines, performed the BR feeding studies and helped to draft the manuscript. FB developed the LC-HRMS method, performed the BR glucoside analyses and helped to draft the manuscript. SF carried out the analysis of endogenous BR contents. WR performed co-localisation experiments, participated in the analysis of the BR glucoside formation data and supported the coordination of this study. MK and FK performed expression analyses. LE, GSH and $\mathrm{YL}$ participated in the generation of over-expression lines. RS, RK and FEV participated in the coordination of this study. HS synthesized the BR-glucosides. DB participated in the design and coordination of this study and helped to draft the manuscript. BP conceived the study, participated in its design and coordination, wrote the manuscript and performed experimental work, such as the generation and analysis of over-expression and GUS reporter lines. All authors read and approved the final manuscript.
Received: 25 November 2010 Accepted: 24 March 2011

Published: 24 March 2011

1. Clouse S: Brassinosteroids. In The Arabidopsis Book. Edited by: Somerville C, Meyerowitz EM. Rockville, MD, USA: American Society of Plant Biologists; 2001.

2. Fujioka S, Yokota T: Biosynthesis and metabolism of brassinosteroids. Ann Rev Plant Biol 2003, 54:137-164.

3. Kinoshita T, Cano-Delgado A, Seto H, Hiranuma S, Fujioka S, Yoshida S, Chory J: Binding of brassinosteroids to the extracellular domain of plant receptor kinase BRI1. Nature 2005, 433:167-171.

4. Kim TW, Wang ZY: Brassinosteroid Signal Transduction from Receptor Kinases to Transcription Factors. Ann Rev Plant Biol 2010, 61:681-704.

5. Choe S: Signal-transduction pathways toward the regulation of brassinosteroid biosynthesis. J Plant Biol 2007, 50:225-229.

6. Neff MM, Nguyen SM, Malancharuvil EJ, Fujioka S, Noguchi T, Seto H, Tsubuki M, Honda T, Takatsuto S, Yoshida S, Chory J: BAS1: A gene regulating brassinosteroid levels and light responsiveness in Arabidopsis. Proc Nat Acad Sci USA 1999, 96:15316-15323.

7. Soeno K, Fujioka S, Hiranuma S, Seto H, Yoshida S: Metabolic Conversion of Castasterone and Brassinolide into Their Glucosides in Higher Plants. J Plant Growth Reg 2006, 25:195-202.

8. Poppenberger B, Fujioka S, Soeno K, George GL, Vaistij FE, Hiranuma S, Seto H, Takatsuto S, Adam G, Yoshida S, Bowles D: The UGT73C5 of Arabidopsis thaliana glucosylates brassinosteroids. Proc Nat Acad Sci USA 2005, 102:15253-15258.

9. Coutinho PM, Stam M, Blanc E, Henrissat B: Why are there so many carbohydrate-active enzyme-related genes in plants? Trends Plant Sci 2003, 8:563-565.

10. Bowles D, Isayenkova J, Lim EK, Poppenberger B: Glycosyltransferases: managers of small molecules. Curr Opin Plant Biol 2005, 8:254-263.

11. Bowles D, Lim EK, Poppenberger B, Vaistij FE: Glycosyltransferases of lipophilic small molecules. Ann Rev Plant Biol 2006, 57:567-597.

12. Lim EK, Higgins GS, Li Y, Bowles DJ: Regioselectivity of glucosylation of caffeic acid by a UDP-glucose:glucosyltransferase is maintained in planta. Biochem J 2003, 373:987-992.

13. Lim EK, Li Y, Parr A, Jackson R, Ashford DA, Bowles DJ: Identification of glucosyltransferase genes involved in sinapate metabolism and lignin synthesis in Arabidopsis. J Biol Chem 2001, 276:4344-4349.

14. Jones P, Messner B, Nakajima J, Schaffner AR, Saito K: UGT73C6 and UGT78D1, glycosyltransferases involved in flavonol glycoside biosynthesis in Arabidopsis thaliana. J Biol Chem 2003, 278:43910-43918.

15. Weis M, Lim EK, Bruce N, Bowles D: Regioselective glucosylation of aromatic compounds: screening of a recombinant glycosyltransferase library to identify biocatalysts. Angew Chem Int Ed Engl 2006, 45:3534-3538.

16. Gandia-Herrero F, Lorenz A, Larson T, Graham IA, Bowles DJ, Rylott EL, Bruce NC: Detoxification of the explosive 2,4,6-trinitrotoluene in Arabidopsis: discovery of bifunctional $\mathrm{O}$ - and $\mathrm{C}$-glucosyltransferases. Plant J 2008, 56:963-974.

17. Poppenberger B, Berthiller F, Bachmann H, Lucyshyn D, Peterbauer C Mitterbauer R, Schuhmacher R, Krska R, Glossl J, Adam G: Heterologous expression of Arabidopsis UDP-glucosyltransferases in Saccharomyces cerevisiae for production of zearalenone-4-O-glucoside. Appl Environm Microbiol 2006, 72:4404-4410.

18. Hou B, Lim EK, Higgins GS, Bowles DJ: N-glucosylation of cytokinins by glycosyltransferases of Arabidopsis thaliana. J Biol Chem 2004, 279:47822-47832.

19. Li Y, Baldauf S, Lim EK, Bowles DJ: Phylogenetic analysis of the UDPglycosyltransferase multigene family of Arabidopsis thaliana. J Biol Chem 2001, 276:4338-4343.

20. Poppenberger B, Berthiller F, Lucyshyn D, Sieberer T, Schuhmacher R, Krska R, Kuchler K, Glossl J, Luschnig C, Adam G: Detoxification of the Fusarium mycotoxin deoxynivalenol by a UDP-glucosyltransferase from Arabidopsis thaliana. J Biol Chem 2003, 278:47905-47914.

21. Vogt $T$, Jones $P$ : Glycosyltransferases in plant natural product synthesis: characterization of a supergene family. Trends Plant Sci 2001, 5:380-386.

22. Schweiger W, Boddu J, Shin S, Poppenberger B, Berthiller F, Lemmens M, Muehlbauer GJ, Adam G: Validation of a candidate deoxynivalenolinactivating UDP-glycosyltransferase from barley by heterologous expression in yeast. Mol Plant Microbe Interact 2010, 23:977-986. 
23. Thummel CS, Chory J: Steroid signaling in plants and insects-common themes, different pathways. Genes Dev 2001, 16:3113-3129.

24. Turk EM, Fujioka S, Seto H, Shimada Y, Takatsuto S, Yoshida S, Wang H, Torres QI, Ward JM, Murthy G, Zhang J, Walker JC, Neff MM: BAS1 and SOB7 act redundantly to modulate Arabidopsis photomorphogenesis via unique brassinosteroid inactivation mechanisms. Plant I 2005, 42:23-34.

25. Small I: RNAi for revealing and engineering plant gene functions. Curr Opin Biotechnol 2007, 18:148-53.

26. Schwab R, Ossowski S, Riester M, Warthmann N, Weigel D: Highly specific gene silencing by artificial microRNAs in Arabidopsis. Plant Cell 2006, 18:1121-1133.

27. Kim GT, Fujioka S, Kozuka T, Tax FE, Takatsuto S, Yoshida S, Tsukaya H: CYP90C1 and CYP90D1 are involved in different steps in the brassinosteroid biosynthesis pathway in Arabidopsis thaliana. Plant $J$ 2005, 41:710-721.

28. Mathur J, Molnar G, Fujioka S, Takatsuto S, Sakurai A, Yokota T, Adam G, Voigt B, Nagy F, Maas C, et al: Transcription of the Arabidopsis CPD gene, encoding a steroidogenic cytochrome P450, is negatively controlled by brassinosteroids. Plant J 1998, 14:593-602.

29. Manabe Y, Tinker N, Colville A, Miki B: CSR1, the sole target of imidazolinone herbicide in Arabidopsis thaliana. Plant Cell Physiol 2007, 48:1340-1358.

30. Baerson SR, Sanchez-Moreiras A, Pedrol-Bonjoch N, Schulz M, Kagan IA, Agarwal AK, Reigosa MJ, Duke SO: Detoxification and transcriptome response in Arabidopsis seedlings exposed to the allelochemical benzoxazolin-2(3H)-one. J Biol Chem 2005, 280:21867-21881.

31. Ferrari S, Galletti R, Pontiggia D, Manfredini C, Lionetti V, Bellincampi D, Cervone F, De Lorenzo G: Transgenic expression of a fungal endopolygalacturonase increases plant resistance to pathogens and reduces auxin sensitivity. Plant Phys 2008, 146:669-681.

32. He JX, Gendron JM, Sun Y, Gampala SS, Gendron N, Sun CQ, Wang ZY: BZR1 is a transcriptional repressor with dual roles in brassinosteroid homeostasis and growth responses. Science 2005, 307:1634-1638.

33. Krishnaswamy SS, Srivastava S, Mohammadi M, Rahman MH, Deyholos MK, Kav NN: Transcriptional profiling of pea ABR17 mediated changes in gene expression in Arabidopsis thaliana. BMC Plant Biol 2008, 8:91.

34. Ferrari S, Galletti R, Denoux C, De Lorenzo G, Ausubel FM, Dewdney J: Resistance to Botrytis cinerea induced in Arabidopsis by elicitors is independent of salicylic acid, ethylene, or jasmonate signaling but requires PHYTOALEXIN DEFICIENT3. Plant Phys 2007, 144:367-379.

35. Hart GW, Haltiwanger RS, Holt GD, Kelly WG: Glycosylation in the nucleus and cytoplasm. Ann Rev Biochem 1989, 58:841-74.

36. Nebert DW: Drug-metabolizing enzymes in ligand-modulated transcription. Biochem Pharmacol 1994, 47:25-37.

37. Radominska-Pandya A, Pokrovskaya ID, Xu J, Little JM, Jude AR, Kurten RC, Czernik PJ: Nuclear UDP-glucuronosyltransferases: identification of UGT2B7 and UGT1A6 in human liver nuclear membranes. Arch Biochem Biophys 2002, 399:37-48.

38. Hong Z, Zhang Z, Olson JM, Verma DP: A novel UDP-glucose transferase is part of the callose synthase complex and interacts with phragmoplastin at the forming cell plate. Plant Cell 2001, 13:769-779.

39. Kleczkowski K, Schell J, Bandur R: Phytohormone conjugates: Nature and function. Crit Rev Plant Sci 1995, 14:283-298.

40. Lim EK, Bowles DJ: A class of plant glycosyltransferases involved in cellular homeostasis. EMBO J 2004, 23:2915-2922.

41. Jones $P$, Vogt $T$ : Glycosyltransferases in secondary plant metabolism: tranquilizers and stimulant controllers. Planta 2001, 213:164-174.

42. D'Auria JC: Acyltransferases in plants: a good time to be BAHD. Curr Opin Plant Biol 2006, 9:331-340.

43. Yu XH, Gou JY, Liu CJ: BAHD superfamily of acyl-CoA dependent acyltransferases in Populus and Arabidopsis: bioinformatics and gene expression. Plant Mol Biol 2009, 70:421-442.

44. Kionka $\mathrm{C}$, Amrhein $\mathrm{N}$ : The enzymatic malonylation of 1aminocyclopropane---carboxylic acid in homogenates of mung-bean hypocotyls. Planta 1984, 162:226-235.

45. Clough SJ, Bent AF: Floral dip: a simplified method for Agrobacteriummediated transformation of Arabidopsis thaliana. Plant J 1998, 16:735-743.

46. Lincoln C, Britton JH, Estelle M: Growth and development of the axr1 mutants of Arabidopsis. Plant Cell 1990, 2:1071-1080.

47. Jackson RG, Kowalczyk M, Li Y, Higgins G, Ross J, Sandberg G, Bowles DJ: Over-expression of an Arabidopsis gene encoding a glucosyltransferase of indole-3-acetic acid: phenotypic characterisation of transgenic lines. Plant J 2002, 32:573-583.

48. Rozhon W, Mayerhofer J, Petutschnig E, Fujioka S, Jonak C: ASKtheta, a group-III Arabidopsis GSK3, functions in the brassinosteroid signalling pathway. Plant J 2010, 62:215-223.

49. Fujioka S, Takatsuto S, Yoshida S: An early C-22 oxidation branch in the brassinosteroid biosynthetic pathway. Plant Physiol 2002, 130:930-939.

50. Poppenberger B, Rozhon W, Khan M, Husar S, Adam G, Luschnig C, Fujioka S, Sieberer T: CESTA, a positive regulator of brassinosteroid biosynthesis. EMBO J 2011.

51. Czechowski T, Stitt M, Altmann T, Udvardi MK, Scheible WR: Genome-wide identification and testing of superior reference genes for transcript normalization in Arabidopsis. Plant Physiol 2005, 139:5-17.

52. Yang Y, Li R, Qi M: In vivo analysis of plant promoters and transcription factors by agroinfiltration of tobacco leaves. Plant J 2000, 22:543-551.

doi:10.1186/1471-2229-11-51

Cite this article as: Husar et al:: Overexpression of the UGT73C6 alters brassinosteroid glucoside formation in Arabidopsis thaliana. BMC Plant Biology 2011 11:51.

\section{Submit your next manuscript to BioMed Central and take full advantage of:}

- Convenient online submission

- Thorough peer review

- No space constraints or color figure charges

- Immediate publication on acceptance

- Inclusion in PubMed, CAS, Scopus and Google Scholar

- Research which is freely available for redistribution

Submit your manuscript at www.biomedcentral.com/submit
Ciomed Central 